\title{
Dynamical simulations of magnetically channeled line-driven stellar winds
}

\author{
Asif ud-Doula and Stanley P. Owocki \\ Bartol Research Institute, University of Delaware, \\ Newark, DE 19716, USA
}

\begin{abstract}
We present numerical magnetohydrodynamic simulations of the effect of stellar dipole magnetic fields on line-driven wind outflows from hot, luminous stars. Unlike previous fixed-field analyses, the simulations here take full account of the dynamical competition between field and flow, and thus apply to a full range of magnetic field strength, and within both closed and open magnetic topologies. A key result is that the overall degree to which the wind is influenced by the field depends largely on a single, dimensionless, 'wind magnetic confinement parameter', $\eta_{*}=B_{\mathrm{eq}}^{2} R_{*}^{2} / \dot{M} v_{\infty}$, which characterizes the ratio between magnetic field energy density and kinetic energy density of the wind. For weak confinement $\eta_{*} \leq 1$, the field is fully opened by the wind outflow, but nonetheless for confinements as small as $\eta_{*}=1 / 10$ can have a significant back-influence in enhancing the density and reducing the flow speed near the magnetic equator. For stronger confinement $\eta_{*}>1$, the magnetic field remains closed over a limited range of latitude and height about the equatorial surface, but eventually is opened into a nearly radial configuration at large radii. Within closed loops, the flow is channeled toward loop tops into shock collisions that are strong enough to produce hard X-rays, with the stagnated material then pulled by gravity back onto the star in quite complex and variable inflow patterns. Within open field flow, the equatorial channeling leads to oblique shocks that are again strong enough to produce X-rays, and also lead to a thin, dense, slowly outflowing 'disk' at the magnetic equator. The polar flow is characterized by a faster-than-radial expansion that is more gradual than anticipated in previous 1D flow-tube analyses, and leads to a much more modest increase in terminal speed $(<30 \%)$, consistent with observational constraints. Overall, the results here provide a dynamical groundwork for interpreting many types of observations, e.g., UV line-profile variability; red-shifted absorption or emission features; enhanced density-squared emission; and X-ray emission, that might be associated with perturbation of hot-star winds by surface magnetic fields.
\end{abstract}

\section{General overview of project}

The abstract above is from ud-Doula \& Owocki (2002), the first paper of an extended PhD study of magnetic channeling of line-driven stellar winds.

Hot, luminous, OB-type stars have strong stellar winds that can be fairly well explained by modern extensions (e.g., Pauldrach, Puls \& Kudritzki 1986) of the basic formalism developed by Castor, Abbott \& Klein (1975, hereafter CAK) for wind driving by scattering of the stellar radiation in a large ensemble of spectral lines. There is evidence that these hot-star winds are not spherically symmetric, steady, smooth outflows, as adopted in CAK-type models, but instead have extensive structure and variability on both large and small scales. 
Large scale structures, as evidenced by explicit UV line-profile variability, are likely to be the consequence of wind processes occurring in the underlying star. E.g., radial and/or non-radial pulsations can cause wind modulation leading to large scale variability in UV lines. Alternatively, magnetic fields can perturb and even channel the wind outflow, leading to rotational modulation of wind structure. This is the possibility we explore in this work.

\section{Results}

Using the publicly available magnetohydrodynamic (MHD) code ZEUS-3D, we study the competition between the magnetic field and the wind. We assume ideal MHD conditions and an isothermal wind. We evolve our azimuthally symmetric 2D MHD simulations from an initial condition, when a dipole magnetic field is introduced into a previously relaxed, one-dimensional, spherically symmetric CAK wind. For the simplified case of isothermal outflow from a non-rotating star, the key results of these simulations are summarized in the abstract. Generally, we find that the degree to which the wind is influenced by the magnetic fields depends largely on a dimensionless, 'wind magnetic confinement parameter', $\eta_{*}=B_{e q}^{2} R_{*}^{2} / M v_{\infty}$.

Recently, we have extended this study to include field-aligned rotation. We find that the rotation plays an important role when it is a significant fraction of 'critical' velocity (defined when outward centrifugal force due to rotation equals the inward gravity). For strong magnetic confinement, the maximum extent of the closed magnetic loops can be limited by the co-rotation radius. The spin-up of the wind material building at the top of the loops leads to the increase of the outward centrifugal pressure, eventually allowing the material to break through the closed field lines and be flung away. These dense slowly outflowing knots may provide a model for discrete absorption components seen in UV wind lines.

In the idealization of isothermal winds, we assumed that hot gas heated up in a shock radiates away its energy instantaneously. The other extreme of this scenario is the adiabatic case, assuming absolutely no radiative losses. We have done some preliminary studies of such winds and find that it leads to configurations that differ substantially from the isothermal models. For example, in the adiabatic case, even modest magnetic confinement, $\eta_{*} \simeq 1$, can trap low density hot gas within closed magnetic loops. In practice, in order to model hotstar winds properly, one needs to consider full energy balance with an accurate cooling function. This is another topic of our current research.

Acknowledgments. This work was supported by the NASA Space Grant College program at the University of Delaware, by NASA grants NAG5-3530 and NAG-11095, and by NSF grant AST-0097983.

\section{References}

Castor, J.I., Abbott, D.C., Klein, R.I. 1975, ApJ 195, 157 (CAK)

Pauldrach, A.W.A., Puls, J., Kudritzki, R.-P. 1986, A\&A 164, 86

ud-Doula, A., Owocki, S.P. 2002, ApJ 576, 413 